\title{
AN ANATOMICAL AND SURGICAL STUDY OF THE EXTRA-THORACIC FASCIA
}

\author{
BY \\ MICHEL LATARJET AND PIERRE JUTTIN \\ From the Anatomy Department of the Faculty of Medicine of Lyons and the National Institute of Hygiene
}

(RECEIVED FOR PUBLICATION MARCH 6, 1953)

Under the title of "Fascia Exo-Thoracique," Gabrielle, Latarjet, and Michaud (1947) have described an extremely thin, homogeneous, and continuous fascial layer which covers the surface of the thoracic cage, posteriorly and laterally. This is a well-known surgical fact and is frequently used in suturing the resulting costo-intercostal incisions of thoracotomies. However, the literature does not effectively describe this fascia either in the sense of its possessing recognizable morphological characteristics, or those histological properties which render valuable service to the thoracic surgeon.

The classical papers, French, English, American or German, describe a plane of cleavage interposed between a posterior muscular plane (levator scapulae, rhomboids, and serratus magnus) and the costo-intercostal surface anteriorly. Furthermore, this space was supposedly brought into existence by the incessant movements of the scapula upon the thoracic wall. This gave birth to the idea of a pseudo-articulation which was described and called the "l'articulation scapulothoracique" by Miramond de Laroquette (1909). It has since remained the classical description.

This study will show that the interposed space is bounded by two layers: one is posterior and covers the anterior surface of the rhomboids and the serratus magnus; the second is anterior and rests upon the costo-intercostal surface. This second layer will henceforth be referred to as the "extra-thoracic fascia" by analogy with the "endo-thoracic fascia" which is interposed between the parietal pleura and the costo-intercostal surface.

\section{Anatomical Procedures}

If the posterior integuments, the trapezius and latissimus dorsi are reflected exposing the levator scapulae, the rhomboids, and the scapula, it is seen that the scapula is covered by the supra- and infraspinatus and their aponeuroses. It is prolonged inferiorly by the inferior portion of the serratus magnus. The rhomboids are reflected medially to their spinal origins by cutting the muscle at its scapular insertions. In order to obtain a better view of the region, it is necessary to reflect laterally the scapula with its attached muscles, thus exposing the entire posterior region of the thoracic cage. In the median plane are the outlines of the transverse vertebral processes and the heads of the ribs, irrespective of the deep muscles of the back. Slightly laterally, the fibres of the serratus muscles, posterior, superior, and inferior, reunited by the aponeuroses of the deep muscles, are seen. However, the latter muscles and the aponeuroses are themselves covered by a recognizable fascial layer, extremely thin and transparent medially, thicker, with minute, irregular eruptions of adipose tissue laterally: this is the extra-thoracic fascia.

\section{BOUNDARIES}

The boundaries are illustrated in Figs. 1, 2, 3, and 4.

Medial.-The medial limit originates in the region of the vertebral attachments of the rhomboid and latissimus dorsi, generally extending only to the beginning of the ninth thoracic vertebra.

LATERAL.-The lateral limit merges at the anterior insertions of the serratus anterior, that is, the fascia, beginning at the lateral portion of the second rib, descends obliquely downwards and anteriorly towards the mid-axillary line, which it reaches at the eighth rib ; the lateral limit extends lower than the medial limit.

These limits, lateral and medial, mark the route by which the extra-thoracic fascia merges with the more superficial aponeuroses (Fig. 1). Thus, in the region of origin of the rhomboid (medial limit of the fascia) there is a continuation with the aponeurosis covering the anterior surfaces of the rhomboid, which itself is continuous with that of the serratus magnus. Fig. 1 demonstrates this 
Fig. 1.-Horizontal section of the thoracic wall (sixth dorsal vertebra). (1) Latissimus dorsi; (2) rhomboid; (3) iliocostalis; (4) serratus dorsalis cranialis; (5) serratus anterior; (6) subscapularis; (7) infraspinalis. The interrupted line marks the route by which the extra-thoracic space (8) merges with the more superficial aponeurosis.

Fig. 2.-Sagittal section of the thoracic wall, through the scapula. (1) Trapezius; (2) supraspinalis; (3) infraspinalis; (4) latissimus dorsi; (5) serratus anterior; (6) subscapularis; (7) aponeurosis of the serratus anterior; (8) extra-thoracic fascia.

Fig. 3. Sagittal section of the thoracic wall medial to the scapula. (1) Trapezius; (2) latissimus dorsi; (3) rhomboid; (4) levator scapulae; (5) extra-thoracic fascia.

Fia. 4. -Sagittal section of the thoracic wall, medial, and inferior to the scapula. (1) Trapezius; (2) latissimus dorsi; (3) extrathoracic fascia. The crosses show, upwards, the extra-fascial space, downwards and anterior, the sub-fascial space.

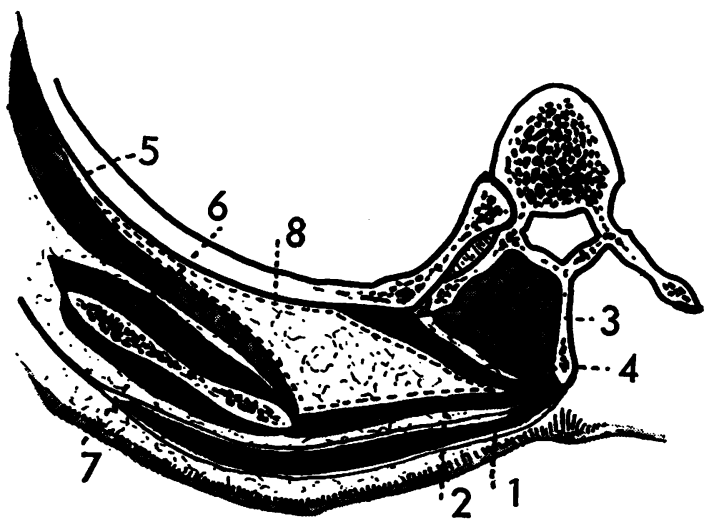

Fig. 1.

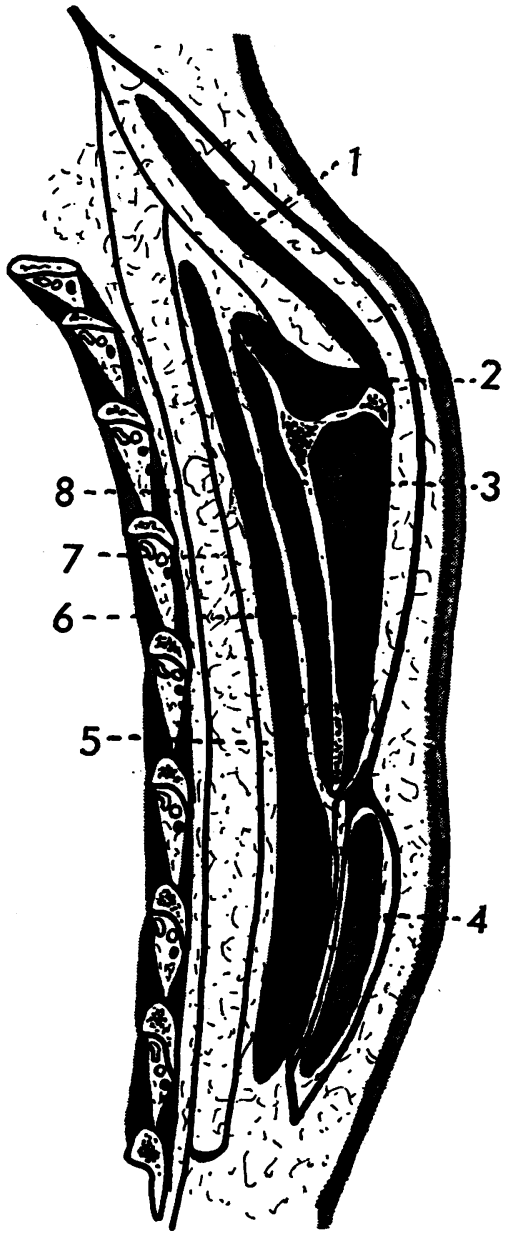

Fig. 2.

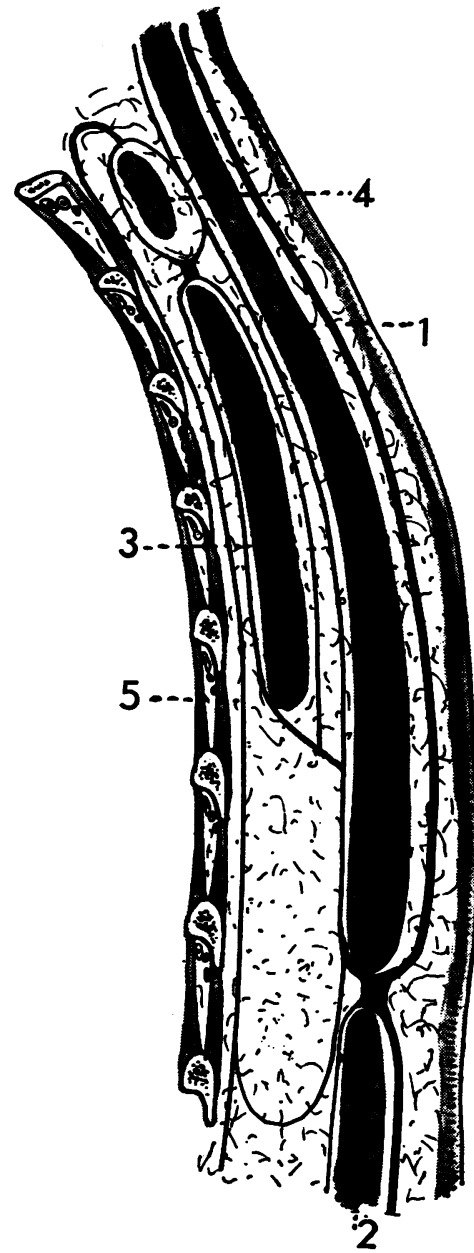

Fig. 3.

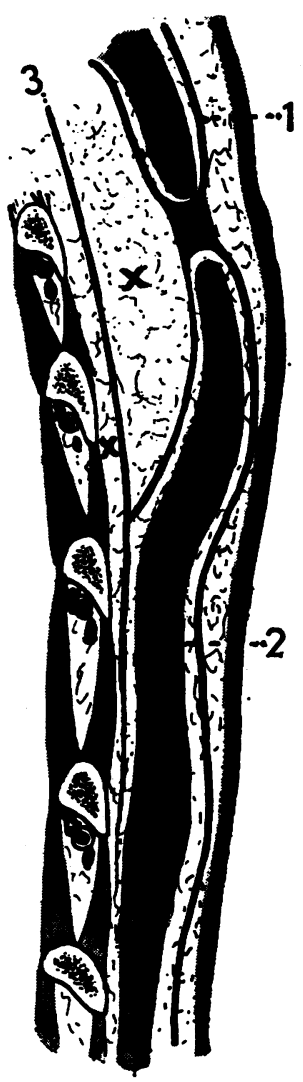

Fig. 4. 
continuity, as well as the space between them which separates the anterior muscles of the scapula from the thoracic cage. The practical and anatomical importance of this space will be discussed later.

SUPERIOR.-At first glance, the extra-thoracic fascia appears to follow the posterior surface of the scalenus posterior, but in reality, and dissection demonstrates this very clearly, the fascia lies as follows.

Above the scapula it merges superiorly and posteriorly with the aponeurosis of the trapezius (Fig. 2). Medially to the scapula the fibres run upwards and posteriorly to merge with the aponeurosis enveloping the levator scapulae (Fig. 3). Thus, in addition to the closing off of the space medially and laterally by the medial and lateral limits of the extra-thoracic fascia, its fusion with the aponeurosis of the trapezius and the levator scapulae limits the space superiorly.

INFERIOR.-The inferior border of the extrathoracic fascia runs parallel to the body of the ninth rib or, less frequently, the ninth intercostal space. Thus its course is obliquely downwards and lateral and it is covered by the anterior surface of the latissimus dorsi medially and of the serratus magnus laterally. The inferior border is more complex, and here the extra-thoracic fascia divides into two sheets (Fig. 4): (1) the deep sheet, which is thinner than the superficial, merges with the thoraco-lumbar region; (2) the superficial sheet, more resistant, unites with the anterior part of the aponeurosis of the latissimus dorsi medially and that of the serratus magnus laterally. Thus union with these different aponeuroses inferiorly closes the infero-posterior line of the retro-thoracic space. By assimilating the other limits this space appears as an irregular, enclosed space.

\section{MORPHOLOGY}

The extra-thoracic fascia should be studied on the living man or the fresh cadaver. Injected specimens cause this fascia to lose individuality and it becomes confused with its underlying structures. In the living body, the extra-thoracic fascia is easily identified by its characteristic appearance. Its composition is not homogeneous, being thin superiorly (on the posterior surface of the scalenus and the superior digitations of the serratus magnus), it thickens from above downwards, a thickening caused by a lamination of layers. The thickening can be seen at thoracotomy which causes a change in the texture of the fascia. Before the incision the fascia blends with the surrounding elements, but several minutes after- wards the fascia condenses and thickens, offering a solid grip to the forceps. Another property, which manifests itself on incision, is its elasticity. It is shown by the enlargement of the space between the two lips of the incision, which seem to disappear under the neighbouring muscles.

The extra-thoracic fascia is a strong membrane $\overrightarrow{0}$ and this has an important surgical bearing. After $\overrightarrow{\vec{H}}$ developing to its maximum thickness between the $\vec{\omega}$ fifth and eighth ribs, the fascia thins out again in the region of the ninth rib. The splitting, which $\times$ was described previously, further decreases its $\stackrel{\infty}{\oplus}$ thickness, resulting in a membraneous appearance. i This membrane is not to be considered a part of $\stackrel{\infty}{N}$ the extra-thoracic fascia but a prolongation of the 은 lumbar aponeurosis. Medially, in the region of the deep muscles of the back, the fascia is thin. However, on its way to the lateral aspect of the thoracic wall, it thickens quickly to its maximum, which it maintains anteriorly.

\section{Microscopic ANATOMY}

Sections taken from different regions were examined histologically for their constituent elements and their relationship to neighbouring fascia. Impressed by the elasticity of the extrathoracic fascia in vivo, the sections were stained with Weigert-safran as well as with haematoxylin $\stackrel{\square}{\square}$ and eosin.

CONSTITUENT ELEMENTS.-The extra-thoracic $\stackrel{0}{3}$ fascia is composed of fatty connective tissue (ring- $?$ shaped), well vascularized, and rich in elastic fibres. The blood vessels are numerous posteriorly, but relatively rare near the surface normally in contact with the thoracic cage. The elastic fibres are dis- $\tilde{x}$ posed singly and in bundles, and, some long and ${ }_{0}$ thin, others short and squat, tend to orientate themselves parallel to the laminae of the fascia, but $ᄋ$ each differs in direction from the neighbouring laminae (Fig. 5).

The histological arrangement explains the solidity and elasticity of the extra-thoracic fascia. It also explains its reactions to inflammation and suppuration.

Relation to Neighbouring Fasciae.--It is easily deduced that the extra-thoracic fascia $\omega$ lies between two cellular spaces which are brought into existence by its presence (Fig. 6). The spaceso can be divided into the space separating the super- $-\Phi$ ficial muscular plane from the fascia and the space? interposed between the fascia and the thoracic 0 cage. The extra-thoracic fascia is independent and can slide smoothly between the muscles and the osteo-muscular wall. The existing space between fascia and thorax has also an importance medially 


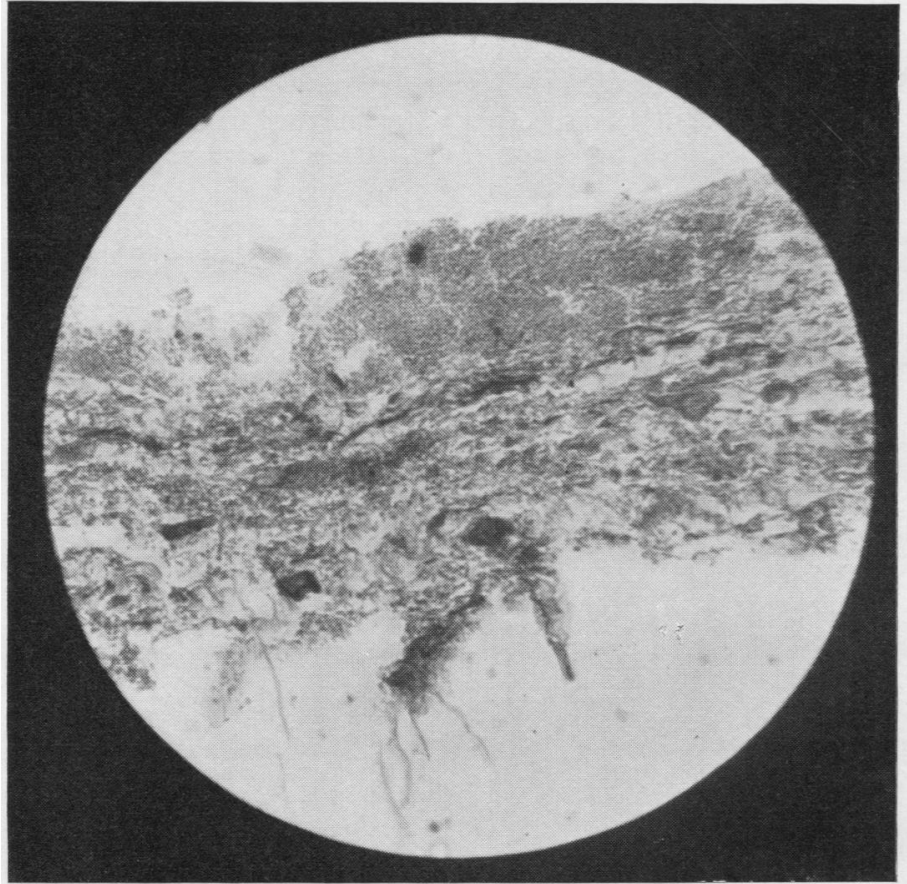

FIG. 5.

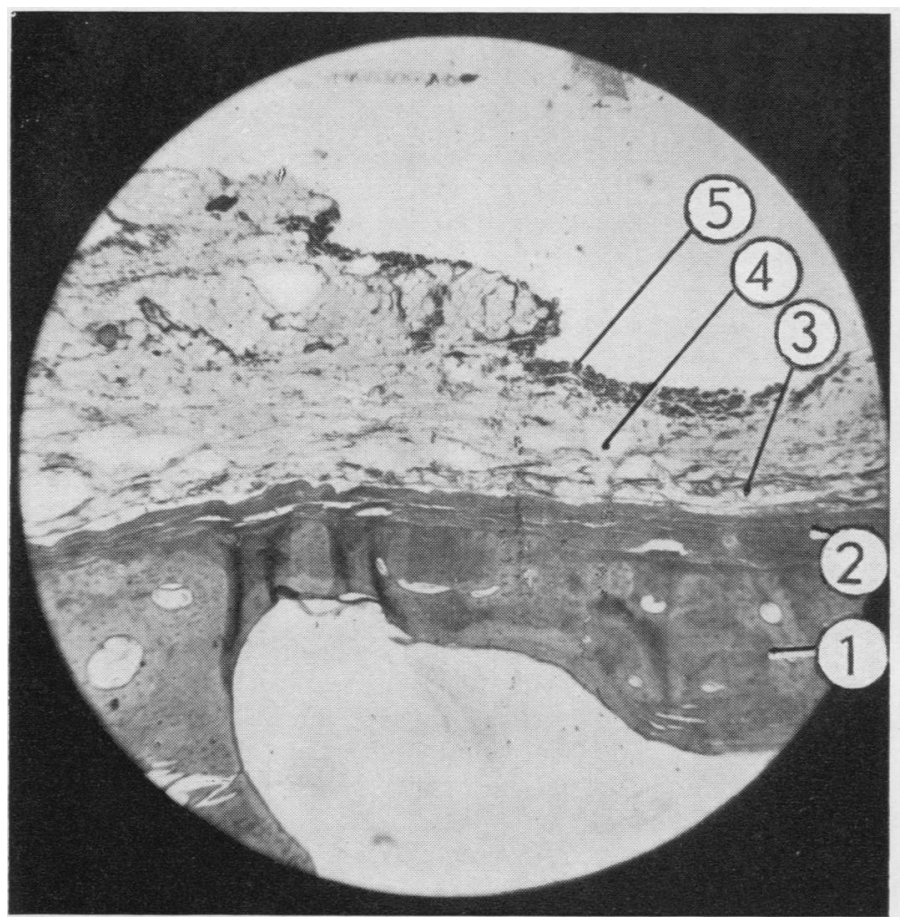

FIG. 6.
FIG 6.-(1) Bone (rib); (2) periosteum; (3) extrathoracic fascia; (4) extra-fascial space; (5) aponeurotic layer.

FIG. 7.-Vertical section of the thoracic wall: the closure of the wound after thoracotomy with rib resection. (1) Parietal pleura; (2) periosteum of the resected rib: (3) extra-thoracic fascia ; (4) muscular laver; (5) skin. The thread which goes through the pleura, the intercostal muscles, and the extra-thoracic fascia closes the sub-fascial space (6). providing extensive emphysema upwards and downwards.

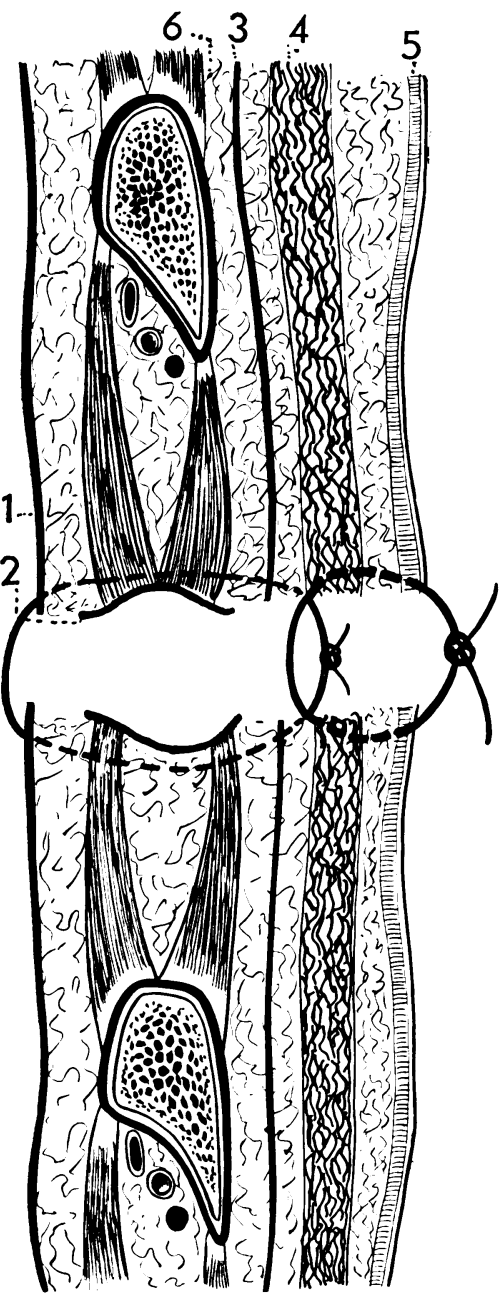

FIG. 7. 


\section{The Posterior Thoracic Spaces}

Depending upon their position, the spaces can be called the extra-fascial space and the sub-fascial space.

Extra-Fascial Space.-This lies between the muscular plan of the levator scapulae, the rhomboid, the serratus magnus on the one hand, and the extra-thoracic fascia on the other. Furthermore, the splitting and junctions of its limits result in a virtually closed pocket of fairly large dimensions (Fig. 1). This can be made evident by lifting up the scapula, exposing a triangular space limited at its summit by the digitation of the serratus magnus supero-laterally and the scalenus posterior supero-medially. The apex is covered posteriorly by the levator scapulae. After passing the superior border of the first rib the apex becomes horizontal or at least strongly oblique in front and above (Fig. 3).

The medial side of the triangle lies against the spinal processes which yield attachment to the rhomboids. It is also superficial to the "deep muscles of the back" and, arriving at the ninth rib, this line forms a curve to join with the basis of the triangle.

The lateral side runs obliquely downwards and forwards following the anterior insertions of the serratus magnus, but, at the eighth rib, changes direction to curve backwards.

The basis is concave from above downwards, hence the border crosses the posterior axillary line in the region of the ninth rib. Here, the serratus magnus has already disappeared and the space forms an inferior "cul de sac" which lies against the anterior surface of the latissimus dorsi. In the extra-fascial space the appearance of the extrathoracic fascia is variable. Between the scapula and the spinal processes the sheet is easily separated. Anterior to the scapula and serratus magnus, a variable mass of connective tissue unites the anterior aponeurosis of this muscle and the extra-thoracic fascia. Miramon de Laroquette has described the various aspects of this space as " seldom free, more often interrupted by connective tissue adhesions which, in order to expose the posterior aspect of the ribs, must be cut by scissors."

Sub-Fascial Space.-This is not a closed space as the above, but simply a plane of cleavage. Hence its communication with the neighbouring spaces is of importance. Laterally and medially the fascia is fastened to the muscles with which it is in contact. Above and below it communicates freely with the following regions. Above it opens into the space beneath the superficial cervicalo aponeurosis, and, by a narrow opening, com-음 municates with the space which separates the first digitation of the serratus magnus from the superior $\mathbb{\Phi}$ fibres of the serratus posterior and superior, ando below it continues into the region beneath the muscles of the thoraco-lumbar region, inferior to. the ninth rib (Spitalier and Aubrespy, 1950).

\section{Surgical Value of the Extra-Thoracic FASCIA}

Closure of The ThoraX.-The gap of a thora cotomy must be sealed hermetically. Parietal emphysema, mediastinal disequilibrium, inabilityo to maintain pulmonary collapse after an extrapleural pneumolysis, are some of the consequences of any suture which allows air to escape. $\stackrel{\mathbb{D}}{\mathbb{N}}$ Different procedures are required after a thoracotomy with or without costal resection.

Thoracotomy without Costal Resection.-Oneor more ribs are sectioned posteriorly, and to closec the resulting gap it is necessary to approach the $\mathrm{e}^{\omega}$ adjacent ribs in order to suture the intercostalo muscles. The orientation of the muscle fibres and the fragility of their aponeurosis render this sutureo precarious. To reinforce it the surgeon oftenô binds the two adjacent ribs. The intercostal nerves 2 cannot support this trauma without pain. In order $\vec{F}$ to prevent this pain and yet obtain a sound suture, 3 the surgeon must use other methods, and we pro-? pose reinforcement by the extra-thoracic fascia.

Thoracotomy with Costal Resection.-In this case, the suture is supported by the costal peri-응 osteum which was carefully guarded during thex osseous resection. However, at times it is damaged by the blades of the retractor. Again, in extraperiosteal pneumolysis, its resistance is diminished by the intercostal separation.

Thoracotomy with Temporary Section.-A hinge is produced by leaving the rib attached to the supero-posterior part of the periosteum. This operation gives the rib a strong attachment superiorly, but does not hold it in place laterally. $N$ The superficial muscles do not hold the ribN against the thoracic wall.

Thus, no matter which technique is utilized, the surgeon needs a supplementary suture to ensure? that the gap is closed. Quite often surgeons, without identifying or naming the extra-thoracic fascia, have made use of it to reinforce the primaryo suture.

The extra-thoracic fascia must be used systematically. After first closing the gap, the thread is 0 brought back, the needle sewing the extra-thoracice 
fascia and the intercostal wall together. It appears useless to suture the fascia alone: air which escapes from the primary suture spreads into the subfascial space, and passes into the supra-clavicular region above and the lumbar region below. By contrast, by suturing this fascia as we suggest (Fig. 7) the sub-fascial space is closed and the spread of emphysema prevented. Furthermore, this suture reinforces the coherence of the musculo-periosteal suture. Unfortunately the extra-thoracic fascia is thinnest in the region where it could be most useful, i.e., at the postero-medial angle of the ribs. However, it is possible to make use of the serratus posterior and superior or the levatores costorum, muscles which can be conserved on removal of the rib. Certain surgeons prefer to use a thin section of the rhomboid muscle to reinforce the sutures above the sixth rib. We prefer to use the extrathoracic fascia, which is simpler, more certain, and anatomically correct.

Utilization of the Extra-Fascial Space.This space is, above all, useful in the posterolateral thoracoplasty. On sectioning the trapezius and rhomboid to reflect the scapula, the surgeon exposes this space. After having cut the frequent connective tissue adhesions between the extrathoracic fascia and the aponeurosis of the serratus magnus, he uncovers the costo-intercostal surface covered by the same fascia. After the ribs have been resected, the floor of the space, i.e., the extrathoracic fascia, loses its continuity and adheres to the collapsed lung, forming a concave depression. The depression varies in depth with the extensiveness of the resection. Inevitably, it is in this concavity that blood-stained serum collects. Certain surgeons consider this fluid a valuable counteracting agent against secondary expansion of the lung. However, most operative techniques drain the extra-fascial space, not only to avoid inflammatory fluids but, above all, to obtain as soon as possible a remodelling of the soft tissues covering the collapsed lung. Since the angle of inclination of the space follows the ninth rib, the drainage, to be completely successful, must originate at the eighth rib. In practice, the surgeon fixes the drainage slightly lateral to the posterior axillary line at the outer edge of the highest remaining rib. But, the extra-fascial space reaches lower, between the thoracic wall and the superficial muscles. In such a drainage, the fluids, which tend to descend to the level of the ninth rib, accumulate below the drain. It is impossible to drain the space efficiently at this level, but if the drainage tube is placed close to the lateral axillary line near the anterior part of the eighth rib, free drainage results because of the favourable slope.

Post-operative healing shows that the extrafascial space has closed. Dense connective tissue appears, making it difficult to find the space again in a second-stage thoracoplasty or in an operation for unsuccessful apical collapse. This space is, at times, the seat of suppuration, complications which arise after thoracoplasties or thoracotomies. By making use of the described limits of the extrafascial space, it is possible to follow the evolution of a suppuration and, at the same time, to drain it effectively.

\section{SUMMARY}

The anatomy and microscopy are described of a fascial layer, which is named the "extra-thoracic fascia," between the muscles of the posterior thoracic wall and the costo-intercostal structures.

It is the floor of a closed space made of connective tissue, whose lower level is, backwards, along the ninth rib.

The extra-thoracic fascia covers the sub-fascial space, which communicates freely with the neighbouring regions, i.e., the supra-clavicular region above, and the lumbar region below.

The extra-thoracic fascia, sealed with the costointercostal plane, enforces effectively the closure of thoracotomies.

The extra-fascial space must be recognized, and eventually drained.

\section{REFERENCES}

Gabrielle, H., Latarjet, M., and Michaud, P. (1947). Lyon chir., 42 101 .

Miramond de Laroquette (1909). Rev. Orthop., ser 2, 10, 311.

Spitalier and Aubrespy (1950). Marseille chir., 2, 223. 